\title{
一种高活性甲醇筹基化的共 聚物铑催化剂研究
}

袁国胍潘平来蒋大智

(中国科学院化学研究所, 北京 100080)

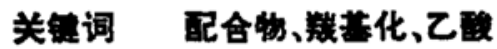

甲醇通过铑催化剂的作用羰基化法制备乙酸, 目前被认为是一种最先进和最经济的方法， 在为数众多的铑催化剂研究中, 以共聚物为配体的催化剂的研究是一个成功先例 ${ }^{[1}$.

我们曾根据 YS 配合方式研究了含有两种不同配位原子的共聚物为配体的铑催化剂 ${ }^{[2]}$, 在 催化甲醇厥基化反应中均表现出很高的催化活性 ${ }^{[3]}$.

本文采用 2-乙烯吡啶 (Y)-丙烯腈 (B) 共聚物为配体, 对其与四羰基二氯二铑形成的配 合物 YBRh 催化甲醇碳基化反应的性能进行了研究和表征.

\section{1 实 验}

共聚物配体及配合物根据文献[3]的方法制备. 配合物的红外光谱和 XPS 数据分别在 VR-10 型红外光谱仪和 Kratos AEIES-300 型能谱仪上测定. 甲醇湠基化反应在 FX-100 型高压釜中进行,产物经气相色谱分析各组分含量.

\section{2 结果与讨论}

\section{1 配体的组成及配合物的表征}

表 1 为共聚物 (YB) 的组成及配合物 YBRh 的铑含量, 从表 1 给出的数据可以看到, 配 合物的铑含量不随其聚合物配体中 B 和 $\mathrm{Y}$ 含量的不同而变化, 这种铑含量的基本恒定, 有利于 研究不同配体配合物性能的差异.

表 1 配体的组成及配合物的铑含量

\begin{tabular}{c|c|c|c}
\hline \multirow{2}{*}{ 配合物 } & \multicolumn{2}{|c|}{ YB 配体 } & YBRh \\
\cline { 2 - 3 } & $\mathrm{Y}(\%)$ & $\mathrm{B}(\%)$ & $\mathrm{Rh}(\%)$ \\
\hline YBRh-1 & 30.2 & 69.8 & 2.73 \\
YBRh-2 & 50 & 50.0 & 2.75 \\
YBRh-3 & 60 & 40.0 & 2.73 \\
YBRh-4 & 74.5 & 25.5 & 2.73 \\
YBRh-5 & 88.5 & 11.5 & 2.74 \\
\hline
\end{tabular}

配合物的 XPS 研究结果表明, 配合物 $N_{1 s}$ 结合能 $(401.1-401.3 \mathrm{eV}$ ) 比配位前的相应 配体的结合能升高, 即在配合物形成过程中伴有 $\mathrm{N}$ 原子上的电荷转移, $\mathrm{N}$ 原子显正电性, 而

1992-05-12 收稿,1993-02-05 收修改稿. 
$\mathrm{Rh} 3 \mathrm{~d} 5 / 2$ 的结合能 $(308.6-308.9 \mathrm{eV})$ 比 $\left[\mathrm{Rh}(\mathrm{CO})_{2} \mathrm{Cl}\right]_{2}$ 的相应结合能 $(310.0 \mathrm{eV})$ 低, 说明 铑离子在配合物形成中作为接受体接受了从 $\mathrm{N}$ 原子来的电荷转移, 形成了 $\mathrm{N} \rightarrow \mathrm{Rh}$ 配价键. 由于2-乙烯吡啶与丙烯腈两种单体在共聚物中为圆规排列, 所以除了少量的七圆或九圆环状 配合单元外,基本配合单元为下列三种八圆环结构:

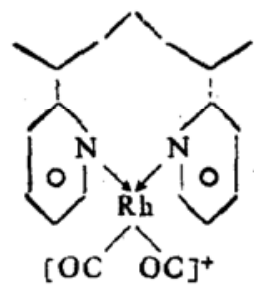

(1)

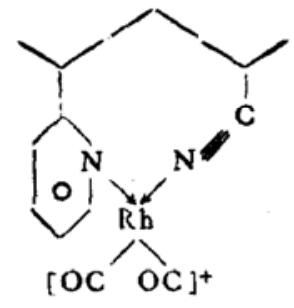

(II)

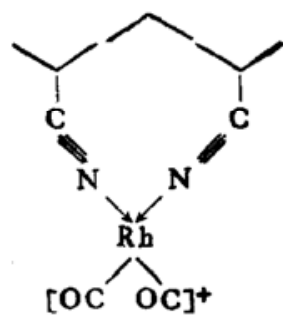

(III)

(I), (II), (III) 单元结构数量随共聚物配体中两种组分含量不同而变化.

上述结构可以从红外光谱的研究得到进一步证明, 在 $2000-2100 \mathrm{~cm}^{-1}$ 处有三组铑的 末 端基吸收峰, 其中结构（I）为 2000,2080 , 结构 (II）为 2030,2100 , 当配合物中铑的含量增多时, 结构 (III) 的铑末端 羓基吸收峰在 $2020,2070 \mathrm{~cm}^{-1}$ 出现, 不同配合物的末端嗾基吸 收峰的吸光度比随配体的组成不同而变化。

另外, YBRh 配合物作为一种高分子催化剂, 其具有优良的 热稳定性. 一般情况下, 顺二羰基铑的配合物对热相对说是很不 稳定的, 例如与铑有较强配位能力的小分子吡啶衍生物和铑的配 合物, 即使在一氧化碳气氮下, 当温度上升为 $160^{\circ} \mathrm{C}$ 以上时, 也会 发生分解而使催化活性明显下降 ${ }^{[4]}$. 图 1 为 YBRh 配合物在空气 中加热时其铑的末端顺二羰基的红外吸收峰变化情况, 如图所示 当温度到 $120^{\circ} \mathrm{C}$ 时, 其 (III) 结构单元的羰基消失, (II) 结构单 元的末端羰基在 $140^{\circ} \mathrm{C}$ 开始逐渐减弱, 即使到 $180^{\circ} \mathrm{C}$ 仍有 (I) 结 构单元的末端羰基存在, YBRh 配合物的这一特点, 是类似的小 分子配合物所不可比拟的.

\section{2 配合物的催化性能}

图 2 为 YBRh 系列配合物催化甲醇羰基化反应的性能与配 体中丙烯腈含量的关系.

如图 2 所示, 配合物的活性随配体中丙烯腈含量的增多而提 高, 这种现象可以从两种 $\mathrm{N} \rightarrow \mathrm{Rh}$ 的强弱得到解释, 铑与吡啶环 上的共轭氮形成的配位键强于与腈基上氮形成的配位键. 结构 （I）的配佂单元虽然稳定, 但活性很低 ${ }^{[9]}$. 结构 (II) 配位单元则 表现出很高的催化活性和稳定性, 配合单元 (III) 虽然具有高的 催化活性, 但其稳定性低于（I）和（II）。这一结论是和图 1 的 三种不同配位单元对热的稳定性的研究结果是吻合的.

在无水条件下, 若以乙酸为溶剂,则加速了反应的进行, 并促 使乙酐的生成。
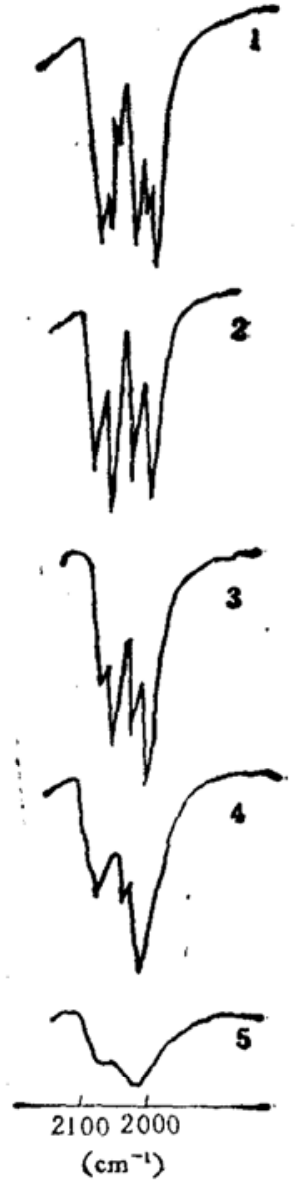

图1 YBRh 配合物末端顺 二䍪基红外吸收峰在不同 温度下变化

1 为加热前, 2 为 $120^{\circ} \mathrm{C}, 3$ 为 $14 n^{\circ} \mathrm{C}, 4$ 为 $160^{\circ} \mathrm{C}, 5$ 为 $180^{\circ} \mathrm{C}$ 


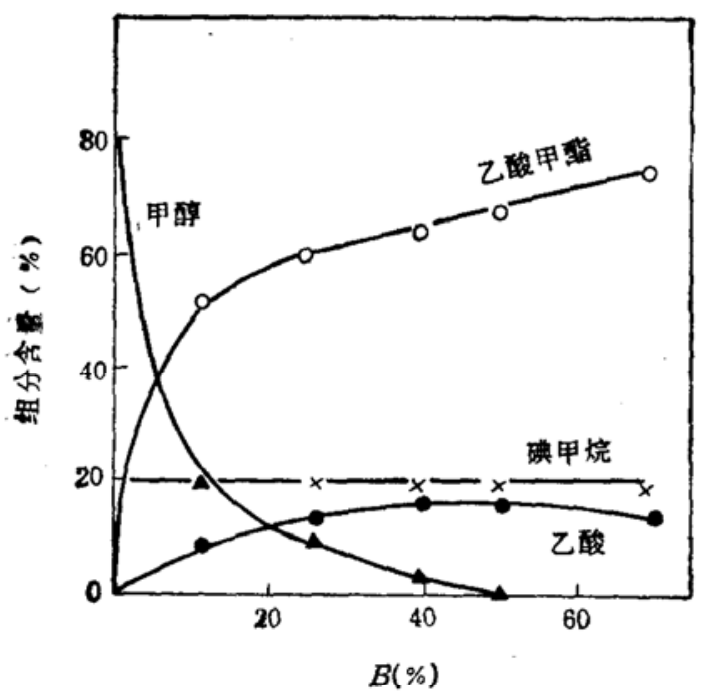

图 2 催化剂的性能与配体中丙烯腈含量的关系 反应条件: 甲醇 $0.49 \mathrm{~mol} ;$ 碰甲烷 $0.08 \mathrm{~mol} ; \mathrm{PCO} 20 \mathrm{~kg} /$ $\mathrm{cm}^{2}$ (初压); R h $6 \times 10^{-3} \mathrm{~mol}$; 温度 $130^{\circ} \mathrm{C}$; 时间 $3 \mathrm{~h}$

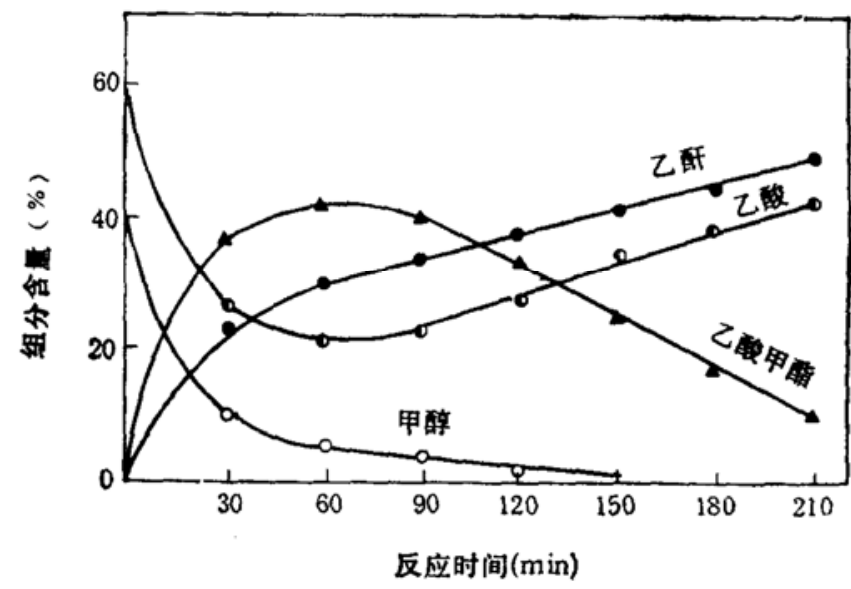

图 3 YBRh-4 配合物催化甲醇-乙酸混合液羰基化 反应条件：甲醇 $0.40 \mathrm{~mol} ;$ 乙酸 $0.2 \mathrm{~mol} ;$ 碘甲烷 $0.08 \mathrm{~mol}$; $\mathrm{PCO} 30 \mathrm{~kg} / \mathrm{cm}^{2}$ (初压);温度 $130^{\circ} \mathrm{C}$; 催化剂用最 $0.2 \mathrm{~g}$

如图 3 所示,在乙酸为溶剂条件下, 在反应初始阶段 (60min 前) 以下列三步反应为主. 首先甲醇与乙酰碘(碘甲烷与一氧化碳反应产物)发生反应:

$$
\mathrm{CH}_{3} \mathrm{OH}+\mathrm{CH}_{3} \mathrm{COI} \rightleftharpoons \mathrm{CH}_{3} \mathrm{COOCH}_{3}+\mathrm{HI}
$$

这一反应从图 3 给出的反应曲线清楚地表现为甲醇含量的降低和乙酸甲酯的升高. 同 时,体系中的乙酸与 $\mathrm{CH}_{3} \mathrm{COI}$ 作用生成乙䣶:

$$
\mathrm{CH}_{3} \mathrm{COOH}+\mathrm{CH}_{3} \mathrm{COI} \rightleftharpoons\left(\mathrm{CH}_{3} \mathrm{CO}\right)_{2} \mathrm{O}+\mathrm{HI}
$$

当反应进行到一定阶段后（60 min 以后）以下列反应为主：乙酸甲酯与(1),(2)式反应 产生的 HI 作用生成乙酸:

$$
\mathrm{CH}_{3} \mathrm{COOCH}_{3}+\mathrm{HI} \rightleftharpoons \mathrm{CH}_{3} \mathrm{COOH}+\mathrm{CH}_{3} \mathrm{I}
$$

乙酤仍按(2)式反应继续增长。

YBRh 配合物催化甲醇羰基化制备乙酸及乙䣶,其反应历程类似于均相反应.

\section{3 结 论}

含有不同配位原子的共聚物为配体的催化剂,在高分子催化剂研究领域形成了独特的“杂 化”配合物催化体系，这种“杂化”配合物具有一般高分子催化剂不可比拟的高活性和通常小 分子催化剂不可比拟的稳定性.

YBRh 系列配合物在温和的条件下，高速度高选择性地催化甲醇羰基化为乙酸及乙䣲。 正是这种“杂化”配合物所具有的优良性能的佐证.

\section{考文姑}

[1] 陈荣耀、袁囷卿、陈予英, 中国专利 (CN1005750B)，1989，11.8.

[2]袁国碞,科学通报, 1987,32(14): 1077 .

[3] Yuan Guo-qing, Chen Yu-ying, Chen Rong-yao, Chiinese Journal of Polymer Scisnce, 1989, 7(3): 219.

[4] Yuan Guo-qing, Chen Rong-yao, Orgamometallic Compounds Symthesis, Structure, and Theory, Texas A\&M Uoiversity Press, College Station, 198.3, 244.

[5] Roth, J. F., Craddock, J. H., Hershman, A. et al., Chsm. Tech., 1971, 600. 